\title{
The Effect of the Asymmetry in the Transport through a Graphene-Based Quantum Structure in Uniform Electric Field
}

\author{
D.M. BĂLTč̆ŢEANU
}

West University of Timisoara, Faculty of Physics, Bd. V. Pârvan, No. 4, RO-300223, Timisoara, Romania

(Received March 22, 2018; in final form April 6, 2019)

The effect of an external and constant electric field on the transmission coefficient and the ballistic conductance is investigated, for a multi-barrier asymmetric quantum structure based on monolayer graphene. It is found that at lower angles of incidence, for certain asymmetric structures the resonant peaks in the transmission gap can be suppressed only by the bias voltage. Furthermore, the pronounced forbidden region that appears in all conductance profiles can be controlled using the external electric field, for an adequate choice of quantum system parameters. Our results could be used for a better description of the transport through such quantum systems and for designing graphene-based devices with potential applications in nanoelectronics.

DOI: 10.12693/APhysPolA.136.55

PACS/topics: graphene, Dirac equation, transmission coefficient, multi-barrier structure

\section{Introduction}

The graphene is one of the most studied materials in the last years [1], due to its unusual and fascinating properties and due to the applications in many fields, especially in nanoelectronics [2-4]. Among other interesting applications, this amazing low-dimensional system can be used in simple experiments for testing quantum electrodynamics effects like the reflectionless Klein tunneling [5]. Graphene is a perfectly two-dimensional (2D) monolayer of carbon atoms, packed in a hexagonal lattice, in which the charge carriers (high mobility electrons and holes with the wave vector $k$ and $-k$, respectively) are treated like relativistic particles without rest mass, due to the interaction with the periodic potential of the lattice [6]. This material is considered as taking part from an extended family that includes single-, bi- and trilayers, each of these members having unique properties and potential applications [6].

In normal conditions, graphene is a gapless "semiconductor" and remains always in the conducting state, this being a major impediment concerning its potential applications for the high-speed optoelectronic devices, especially for digital circuits (logic gates or memories) [7]. However, due to the advances of modern technologies, a band gap can be created and controlled through different experimental procedures [7-9]. In a single-layer graphene a band gap can also be induced and controlled by an external voltage, enabling the formation of gate-tunable potential barriers in which the transport of charge carriers in different conditions can be studied $[2,5,10]$.

There are many interesting studies performed for unbiased graphene-based systems, in which the Klein

e-mail: doru.baltateanu@e-uvt.ro tunneling, the direction-dependent transmission and other quantum phenomena have been revealed [11-18]. Concerning the biased structures, the resonant tunneling has been studied for a double barrier structure, pointing out the effects of the electric field on the conductance [19]. Also, the electrical transport properties in graphene superlattices modulated by a homogeneous electric field have been studied [20].

It is very important to mention that in [20] the current density for a graphene (symmetric) superlattice is computed and comparisons between the monolayer and bilayer graphene structures are performed. Our results are different from [20] because we are focused on the asymmetric graphene structures and the interplay between the electric field and the asymmetry is pointed out.

Motivated by these studies, we propose here a computational model in which the effect of an external and uniform electric field on a multi-barrier quantum structure based on monolayer graphene is investigated. The paper is structured as follows. In Sect. 2 the model for computing the transmission and the conductance through the structure is introduced. The numerical results with discussions are presented in Sect. 3 and the final conclusions are given in Sect. 4.

\section{The theoretical model}

Here we present the model for computing the transmission and the conductance for the ballistic transport at zero-temperature of the charge carries through a multibarrier structure based on monolayer graphene, exposed to an external and constant electric field. Such structures can be obtained experimentally through the (locally and globally) tuning of the charge carriers, using a planar back gate, and a finite number of top gates [21]. A graphene cavity under a certain top gate can be considered as a barrier and the one between two neighboring 
gates as a well, respectively [21]. In addition, to highlight the effect of the external electric field, we consider that any kind of interaction, i.e. charge carriers-phonons interactions or spin-orbit interactions are absent. The honeycomb of the monolayer graphene contains two sublattices $\mathrm{A}$ and $\mathrm{B}$, with two atoms in the unit cell. The conduction and the valence band are connected in six corners of the first Brillouin zone in reciprocal space, creating a gapless energy spectrum $E=E\left(k_{x}, k_{y}\right)$. Only the states around two (non-equivalent) $K$ and $K^{\prime}$ points, corresponding to the Dirac cones that touch each other at the Fermi level $E_{\mathrm{F}}=0$, exhibit a linear dispersion [5,6]. The 2D Dirac Hamiltonian for a relativistic massless fermion at low energies, around the Dirac point, is given by

$$
H=\hbar v_{\mathrm{F}}\left(\sigma_{x} p_{x}+\sigma_{y} p_{y}\right)+I_{2} V(x, y)
$$

in which $\sigma_{x}, \sigma_{y}$ are the Pauli matrices, $p_{x}, p_{y}$ are the components of the quasiparticle momentum, $v_{\mathrm{F}}=c / 300$ is the Fermi velocity in graphene ( $c$ being the speed of light), $V(x, y)$ is the scalar potential, and $I_{2}$ is the $2 \times 2$ identity matrix. Considering that $V(x, y)$ is invariant to translations in the $y$ direction, the wave-vector component $k_{y}$ will be constant and the Dirac equation can be written as

$$
[-\mathrm{i} \hbar \boldsymbol{\sigma} \cdot \nabla+V(x)] \Psi(x, y)=E \Psi(x, y) .
$$

The operator from Eq. (2) acts on the pseudospinors $\psi(x, y)=\left[\psi_{A}(x, y), \psi_{B}(x, y)\right]^{\mathrm{T}}$ with two components, that correspond to the sublattices $A$ and $B$, respectively. Note that for graphene there is not a true electron spin but a degree of freedom called pseudospin. The pseudospins "up" and "down" correspond to the electronic states located to the A and B sublattices, respectively. Due to the translational symmetry in the $y$ direction, one can write the general solutions of the Dirac equation as a combination of incident and reflected plane waves

$$
\begin{gathered}
\Psi_{k, \lambda}(x, y)=\frac{a}{\sqrt{2}}\left(\begin{array}{c}
1 \\
\lambda \mathrm{e}^{\mathrm{i} \phi}
\end{array}\right) \mathrm{e}^{\mathrm{i}\left(k_{x} x+k_{y} y\right)} \\
+\frac{b}{\sqrt{2}}\left(\begin{array}{c}
1 \\
-\lambda \mathrm{e}^{-\mathrm{i} \phi}
\end{array}\right) \mathrm{e}^{\mathrm{i}\left(-k_{x} x+k_{y} y\right)} .
\end{gathered}
$$

In Eq. (3), $a$ and $b$ are complex amplitudes, $\phi$ is the angle of incidence with respect to the growth direction ( $x$-axis), $k_{x}, k_{y}$ are the components of the wave vector along the $x$ and $y$ directions, and $\lambda$ are the eigenvalues of the chirality operator (equivalent with the helicity operator in the case of the real spin). The $\lambda$ values depend on the sign of the energies, $\lambda=\operatorname{sgn}(E-V(x))(\lambda=+1$ for electrons and $\lambda=-1$ for holes). The above solutions will be used for numerically computing the transmission coefficient and the ballistic conductance, using the transfer matrix method for piecewise potentials.

Consequently, we consider a structure with $N$ barriers of widths $L_{b n}(n=1, \ldots N)$ and $N-1$ wells of widths $L_{w p}(p=1, \ldots N-1)$, having the total lengths $L_{x}=L$ and $L_{y}$ along the $x$ and $y$ directions, respectively. We suppose that $L_{y}$ is very large compared to the barriers/wells widths, to neglect the edge effects. The entire domain is divided in a number of $m$ sub-domains of the same (small) length, where the potential is constant.

The electric field is applied along the $x$ axis, between $x_{0}=0$ and $x_{m}=L$. The potential profile for unbiased structure is $V(x)=V_{0}$ for barriers and $V(x)=0$ otherwise. In the presence of a bias voltage the potential is $V(x)=0$ for $x \in(-\infty, 0], V_{0}-\left(V_{a} / L\right) x$ for $x \in\left[x_{j-1}, x_{j}\right)$ with $j=1,2, \ldots m$, and $V(x)=-V_{a}$ for $\left[x_{m},+\infty\right)$, $V_{a}=e U_{a}$ being the potential due to the electric field.

Taking into account the general solutions from Eq. (3), in a certain $j$ sub-domain (barrier or well) we will use vectors of the form

$$
\begin{aligned}
& v_{j}(x, y)=\mathrm{e}^{\mathrm{i} k_{j y} y} \\
& \quad \times\left(\begin{array}{cc}
\mathrm{e}^{\mathrm{i} k_{j x} x} & \mathrm{e}^{-\mathrm{i} k_{j x} x} \\
\lambda_{j} \mathrm{e}^{\mathrm{i}\left(k_{j x} x+\phi_{j}\right)} & -\lambda_{j} \mathrm{e}^{-\mathrm{i}\left(k_{j x} x+\phi_{j}\right)}
\end{array}\right)\left(\begin{array}{c}
a_{j} \\
b_{j}
\end{array}\right),
\end{aligned}
$$

where $\lambda_{j}=\operatorname{sgn}\left[E-V_{j}(x)\right]$ and $j=1,2, \ldots m$.

For a given Fermi energy $E=\hbar v_{\mathrm{F}}|\boldsymbol{k}|$, with the wave vector $|\boldsymbol{k}|=k=\left(k_{x}^{2}+k_{y}^{2}\right)^{1 / 2}$, since $k_{y}$ is conserved we have

$$
k_{j y}=k_{y}=k \sin \phi_{\mathrm{in}}=\left(E / \hbar v_{\mathrm{F}}\right) \sin \phi_{\mathrm{in}},
$$

where $\phi_{\text {in }}$ is the angle at the entrance (IN, $j=0$ ) of the structure. Note that for the biased structure the angle $\phi_{\text {in }}$ is different from the angle $\phi_{\text {out }}$, at the exit region (OUT, $j=m+1$ ).

The wave vectors along the $x$ direction in the $j$ sub-domain ( $k_{j x}^{b}$ for barriers and $k_{j x}^{w}$ for wells) are

$$
\begin{aligned}
k_{j x}^{b} & =\sqrt{\left(E-V_{j b}(x)\right)^{2} / \hbar^{2} v_{\mathrm{F}}^{2}-k_{y}^{2}}, \\
k_{j x}^{w} & =\sqrt{\left(E-V_{j w}(x)\right)^{2} / \hbar^{2} v_{\mathrm{F}}^{2}-k_{y}^{2}},
\end{aligned}
$$

in which $V_{j b}(x)=V_{0}-\left(V_{a} / L\right) x_{j}$ and $V_{j w}(x)=$ $-\left(V_{a} / L\right) x_{j}$ are the potentials in the $j$ domain from a barrier and from a well, respectively, with $x_{j}=x_{j-1}+L / m$. The angle between the direction of propagation and the $x$ axis in the sub-domain $j$ is $\phi_{j}=\phi_{j}^{b}=\tan ^{-1}\left(k_{y} / k_{j x}^{b}\right)$ for a barrier and $\phi_{j}=\phi_{j}^{w}=\tan ^{-1}\left(k_{y} / k_{j x}^{w}\right)$ for a well. The wave-vector components along the $x$ direction for IN/OUT regions are

$$
\begin{aligned}
& k_{(\mathrm{in}) x}=\left(E / \hbar v_{\mathrm{F}}\right) \cos \phi_{\mathrm{in}}, \\
& \quad k_{(\text {out }) x}=\sqrt{\left(E+V_{a}\right)^{2} / \hbar^{2} v_{\mathrm{F}}^{2}-k_{y}^{2}} .
\end{aligned}
$$

The angle $\phi_{\text {in }} \in\left[-90^{\circ}, 90^{\circ}\right]$ and $\phi_{\text {out }}=$ $\tan ^{-1}\left(k_{y} / k_{(\text {out }) x}\right)$. Considering that $a_{0}=a_{\text {in }}=1$, $b_{0}=b_{\text {in }}=r, a_{m+1}=a_{\text {out }}=t$ and $b_{m+1}=b_{\text {out }}=0, r$ and $t$ being the reflection and transmission amplitudes in the IN and OUT domains, respectively, we can compute the total transfer matrix, given by the relation

$$
M_{t}=\left(\prod_{j=1}^{m} M_{j-1 \mid j}\right) M_{m \mid m+1} .
$$

$M_{j-1 \mid j}$ are transfer matrices that connects the vectors $v_{j-1}\left(x_{j-1}, y\right)$ and $v_{j}\left(x_{j-1}, y\right)$ across an interface and the matrix $M_{m \mid m+1}$, which connects the vectors $v_{m}\left(x_{m}, y\right)$ 
and $v_{\text {out }}\left(x_{m}, y\right)$. These matrices are calculated using the boundary conditions (the continuity of the wave functions at the interfaces) and the transmission coefficient can be computed with the expression

$$
T=\left(\cos \phi_{\text {out }} / \cos \phi_{\text {in }}\right)\left(1 /\left|M_{t, 11}\right|^{2}\right),
$$

where $M_{t, 11}$ is the element on the first row/column of the matrix $M_{t}$.

Knowing the transmission coefficient, we can finally calculate the ballistic conductance at zero temperature, using the Landauer-Büttiker expression [22]:

$$
G(E)=G_{0} \int_{-\pi / 2}^{\pi / 2} T\left(E, \phi_{\text {in }}\right) \cos \phi_{\text {in }} \mathrm{d} \phi_{\text {in }},
$$

in which $G_{0}=2 e^{2} E L_{y} /(\pi h)$.

\section{Numerical results and discussion}

In the beginning we present some results concerning the tunneling through a simple $(N=1)$ barrier structure with $L_{b}=100 \mathrm{~nm}$. In Fig. 1a the transmission probability versus the angle of incidence $\phi_{\text {in }}$ is plotted for this barrier, at different bias voltages. The Fermi energy of the incident wave is fixed at the value $E=83 \mathrm{meV}$ (corresponding to a wavelength $\lambda=50 \mathrm{~nm}$ ) and the barrier height is $V_{0}=0.2 \mathrm{eV}$, these representing typical values used in experiments [5]. We can observe from Fig. 1a that the transmission is symmetric with respect to the $x$ axis and for $U_{a}=0 \mathrm{mV}$ our results are in agreement with those of [5]. Without the field, at normal incidence $\left(\phi_{\text {in }}=0\right)$ a perfect transmission occurs, that is a consequence of the Klein tunneling [5]. Also, for angles near to this angle of incidence the barrier remains transparent due to the pseudospin conservation, which ensures the absence of backscattering processes [23]. For a constructive interference of the waves, the Fabry-Pérot transmission resonances occur at angles greater than $30^{\circ}$, the corresponding peaks being very sharp in comparison with the central one. In the presence of the field, the barrier remains perfectly transparent at normal incidence and the transmission profile close to $\phi_{\text {in }}=0$ does not depend on the bias voltage. When the voltage increases (from $10 \mathrm{mV}$ to $50 \mathrm{mV}$ ) the resonance peaks remain sharp but are slowly diminished and shifted towards lower angles.
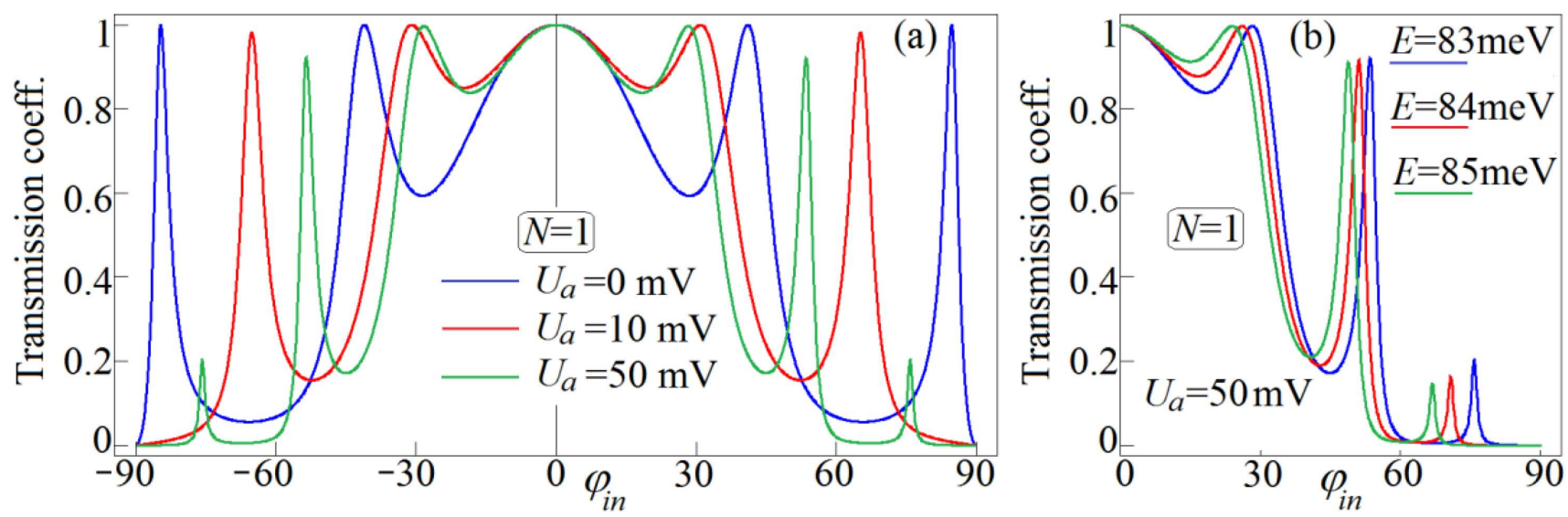

Fig. 1. Transmission coefficient versus the angle of incidence for a single barrier with $L_{b}=100 \mathrm{~nm}$ : (a) $E=83 \mathrm{meV}$ and $U_{a}=0,10,50 \mathrm{mV}$, (b) $U_{a}=50 \mathrm{mV}$ and $E=83,84,85 \mathrm{meV}$.

An interesting observation is that the transmission is very sensitive to a little change of the Fermi incident energy. To see this behavior, in Fig. 1b the angular dependence of the transmission is displayed, for $E=83 \mathrm{meV}$ and other two energies very close to this value $(84 \mathrm{meV}$ and $85 \mathrm{meV}$, respectively) at a constant bias voltage $U_{a}=50 \mathrm{mV}$. Due to the symmetry, the interval for the angles of incidence is restricted to $\left[0^{\circ}, 90^{\circ}\right]$. For a little increase of the energy the peaks are shifted towards lower angles and, in addition, for angles of incidence greater than $30^{\circ}$ they are slowly diminished.

In the following, in Fig. 2 the dependence of the transmission on the incident energy is illustrated for the above barrier, with $V_{0}=0.12 \mathrm{eV}$, at an oblique incidence $\left(\phi_{\text {in }}=10^{\circ}\right.$ - Fig. $2 \mathrm{a}$ and $\phi_{\text {in }}=20^{\circ}$ - Fig. 2b). In this case a gap in the transmission can be observed (due to the evanescent wave modes inside the barrier), which can be modulated by the angle of incidence and the barrier parameters (width and height) $[22,24]$. For the unbiased structure at $\phi_{\text {in }}=10^{\circ}$, our results are in agreement with those from [22]. When $\phi_{\text {in }}$ decreases (in our case from $20^{\circ}$ to $10^{\circ}$ ), the gap becomes narrower, this being also in agreement with [22]. We can observe from Fig. 2 that the narrowing of the gap is maintained for the biased structure. It is interesting that the gap can be also tuned using the external field and this can be clearly seen in Fig. 2, for both angles of incidence. For $\phi_{\text {in }}=10^{\circ}$ and $U_{a}=25 \mathrm{mV}$, the center of the gap is shifted toward lower energies and the width is slowly reduced. If the voltage increases at $U_{a}=50 \mathrm{mV}$, the structure is no more 
$100 \%$ opaque. Due to the external field, the transmission peaks are also shifted towards lower energies and diminished slowly. At the increase of the angle of incidence $\left(\phi_{\text {in }}=20^{\circ}\right)$ the transmission exhibits the same behavior, except the fact that for $U_{a}=50 \mathrm{mV}$ the gap persists, even if the width is reduced. Therefore, the variation of the external field, together with an adequate change of the angle of incidence and geometric parameters of the quantum system, could have potential applications in the area of the graphene-based optoelectronic devices.
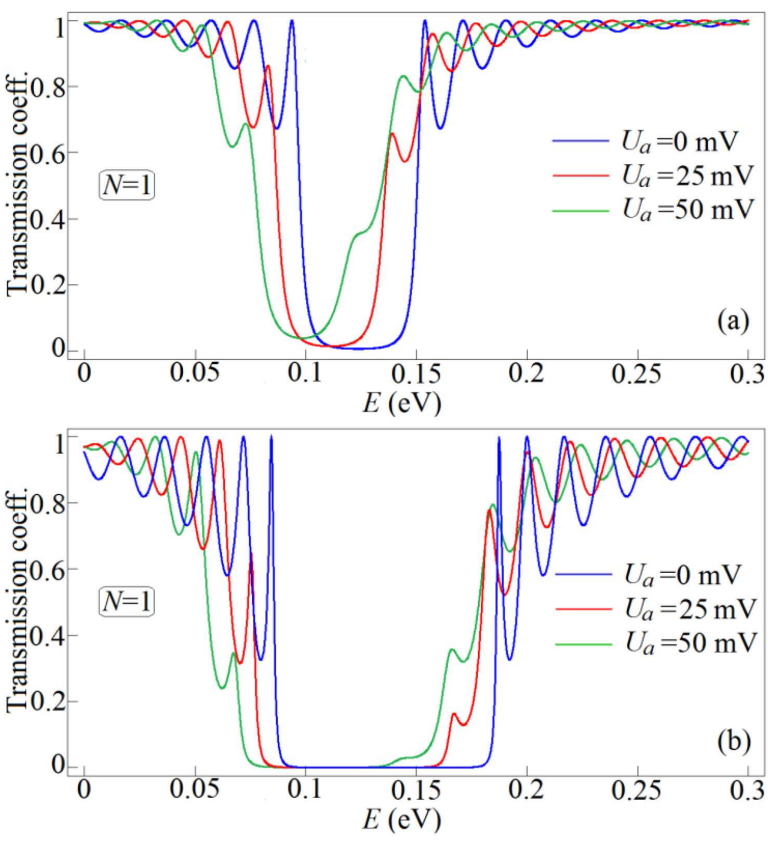

Fig. 2. Transmission versus the energy for a single barrier, at two different angles of incidence, $\phi_{\text {in }}=10^{\circ}$ (a) and $\phi_{\text {in }}=20^{\circ}(\mathrm{b}), L_{b}=100 \mathrm{~nm}, V_{0}=0.12 \mathrm{eV}$, the bias voltage is $U_{a}=0,25$, and $50 \mathrm{mV}$.

Next we focus on a topic related to the apparition of the line-type resonance peaks in the transmission gap (due to the coupling between wells and barriers), that depends on the angle of incidence and the structure parameters. Such peaks are strongly suppressed in the unbiased asymmetric double barrier structures and this could be used in applications with resonant tunneling devices and energy filters [24]. Therefore, we will discuss the apparition of these peaks for symmetric and asymmetric multibarriers with $N=2,3$, and 5 , in the absence/presence of the external field. In Fig. 3 the transmission versus energy is plotted for a symmetric (Fig. 3a and b) and asymmetric (Fig. 3c and d) structure with $N=2$, at $V_{0}=0.2 \mathrm{eV}$ and $\phi_{\text {in }}=10^{\circ}$.

For the unbiased symmetric structure (Fig. 3a), when the incident energy values match the resonant energy in the well, four line-type peaks (with a transmission 100\%) appear in the gap region. We mention that all peaks have been obtained using an energy step $\Delta E=10^{-6} \mathrm{eV}$ in the transmission gap region. In the presence of the electric field, for $U_{a}=50 \mathrm{mV}$, a suppression of the peaks can be observed (Fig. 3b), shifted towards lower energies. For the unbiased asymmetric structure (Fig. 3c) a suppression of the resonances can be observed, which is in agreement with the results from [24]. This suppression becomes more pregnant in the presence of the external field, as we can observe from Fig. 3d. Therefore, at oblique incidence, for an adequate choosing of the multibarrier parameters, the transmission gap can be tuned using the external electric field. Moreover, in the presence of the field, the suppression and also a shift of the line-type peaks can be obtained for the symmetric double barrier structures. This type of gap tuning could also be useful for potential applications of graphene-based devices.

With increasing number of wells, the number of the transmission peaks in the gap region increases, too. For $N$ barriers, there is an $(N-1)$-fold splitting of every peak, similarly with the splitting in the case of electronic transport through semiconductor superlattices [25]. Such kind of splitting has been revealed in magnetic Kronig-Penney graphene superlattices [26], graphene superlattices with one-dimensional periodic potentials [27] or with rectangular electric potential and $\delta$-function magnetic potential [28]. This behavior is also present in our numerical calculations and we show in Fig. 4 the transmission profiles for two asymmetric structures with $N=3$ and $N=5$, for $V_{0}=0.2 \mathrm{eV}$ and $\phi_{\text {in }}=10^{\circ}$.

For $N=3$ (2 quantum wells) each line-type peak is split into 2 peaks, resulting a total number of 8 peaks. For $N=5$ (4 quantum wells) it results in 16 peaks which are very close and cannot be observed in Fig. 4. Therefore, they are illustrated in Fig. 5, in which only the gap region is selected (between $1.82 \mathrm{eV}$ and $2.24 \mathrm{eV}$ ). For a better view, some energy sub-domains in which $T=0$ are omitted (symbolized with dashed lines in Fig. 5).

It must be pointed out that for selected asymmetric structures, the resonance peaks are no more suppressed in the absence of the electric field (Fig. 4a and c). Only in the biased structures they are suppressed and the transmission gap is moved towards lower energies, with the degree of suppression depending on the value of the external electric field. This effect can be seen in Fig. $4 \mathrm{~b}(N=3)$ and Fig. $4 \mathrm{~d}(N=5)$ for a bias voltage $U_{a}=100 \mathrm{mV}$ and suggests again that one can modulate the gap region of the asymmetric structures by varying the external field. The value of the field is an important parameter because in combination with other parameters (the number of the wells and the angle of incidence), energy domains in which the structure is totally transparent or opaque can be obtained. This effect could be used for tuning the transmission in digital quantum circuits based on graphene.

In the following the ballistic conductance at zero temperature is computed for a simple barrier and for asymmetric multiple-barriers with $N=2$ and 5 , respectively. Figure 6 shows this conductance as a function of the incident energy (parts (a)-(c)) and of the potential height $V_{0}($ part $(\mathrm{d}))$, respectively. 


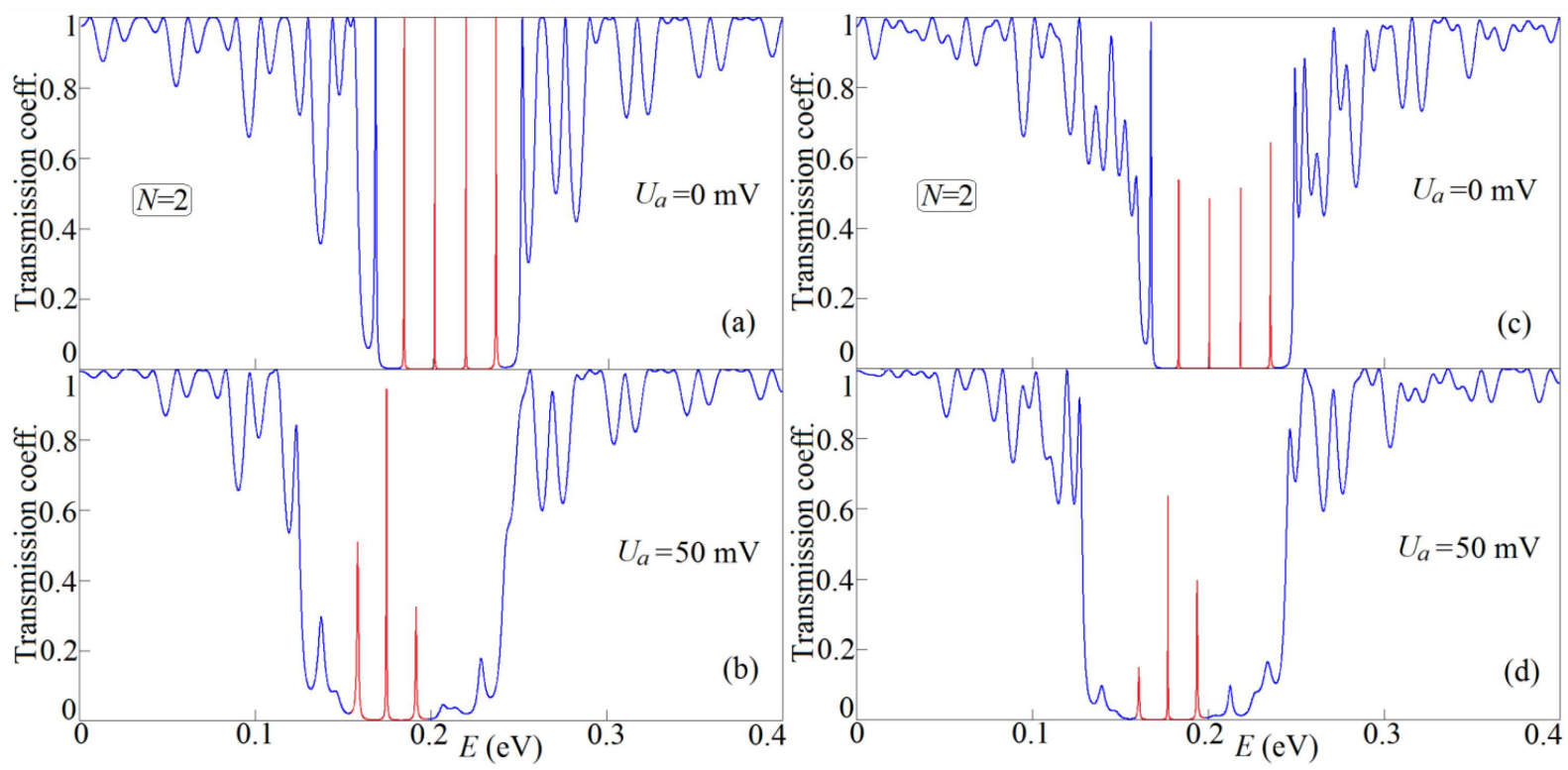

Fig. 3. Transmission versus the incident energy for $N=2$ : (a, b) symmetric structure, $L_{b 1}=L_{b 2}=50 \mathrm{~nm}, L_{w}=$ $100 \mathrm{~nm},(\mathrm{c}, \mathrm{d})$ asymmetric structure, $L_{b 1}=50 \mathrm{~nm}, L_{w}=100 \mathrm{~nm}, L_{b 2}=70 \mathrm{~nm}, V_{0}=0.2 \mathrm{eV}, \phi_{\text {in }}=10^{\circ}$.

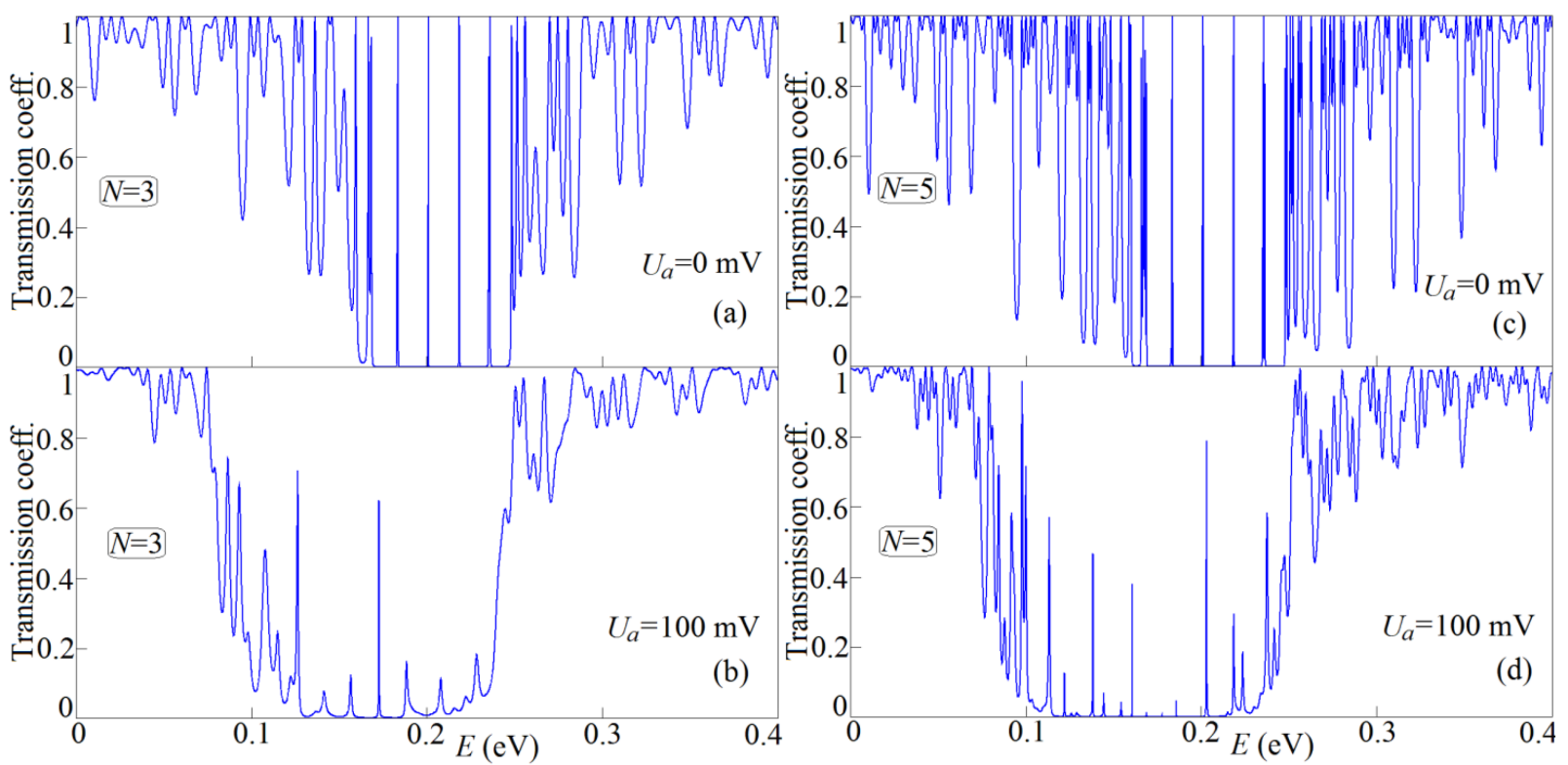

Fig. 4. Transmission coefficient versus the incident energy for two asymmetric structures with $N=3$ (a, b) and $N=5$ (c, d); for $N=3, L_{b 1}=L_{b 3}=50 \mathrm{~nm}$ and $L_{b 2}=70 \mathrm{~nm}$; for $N=5, L_{b 1}=L_{b 3}=L_{b 5}=50 \mathrm{~nm}$ and $L_{b 2}=L_{b 4}=70 \mathrm{~nm}$; the wells lengths are the same in both structures, $L_{w}=100 \mathrm{~nm} ; V_{0}=0.2 \mathrm{eV}, \phi_{\mathrm{in}}=10^{\circ}$.

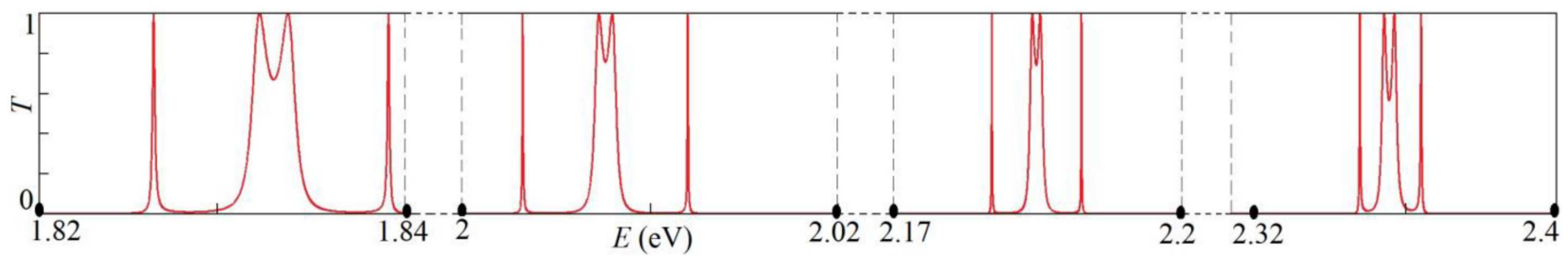

Fig. 5. Splitting of the resonance peaks for the asymmetric structure with $N=5$, from Fig. 4 . 

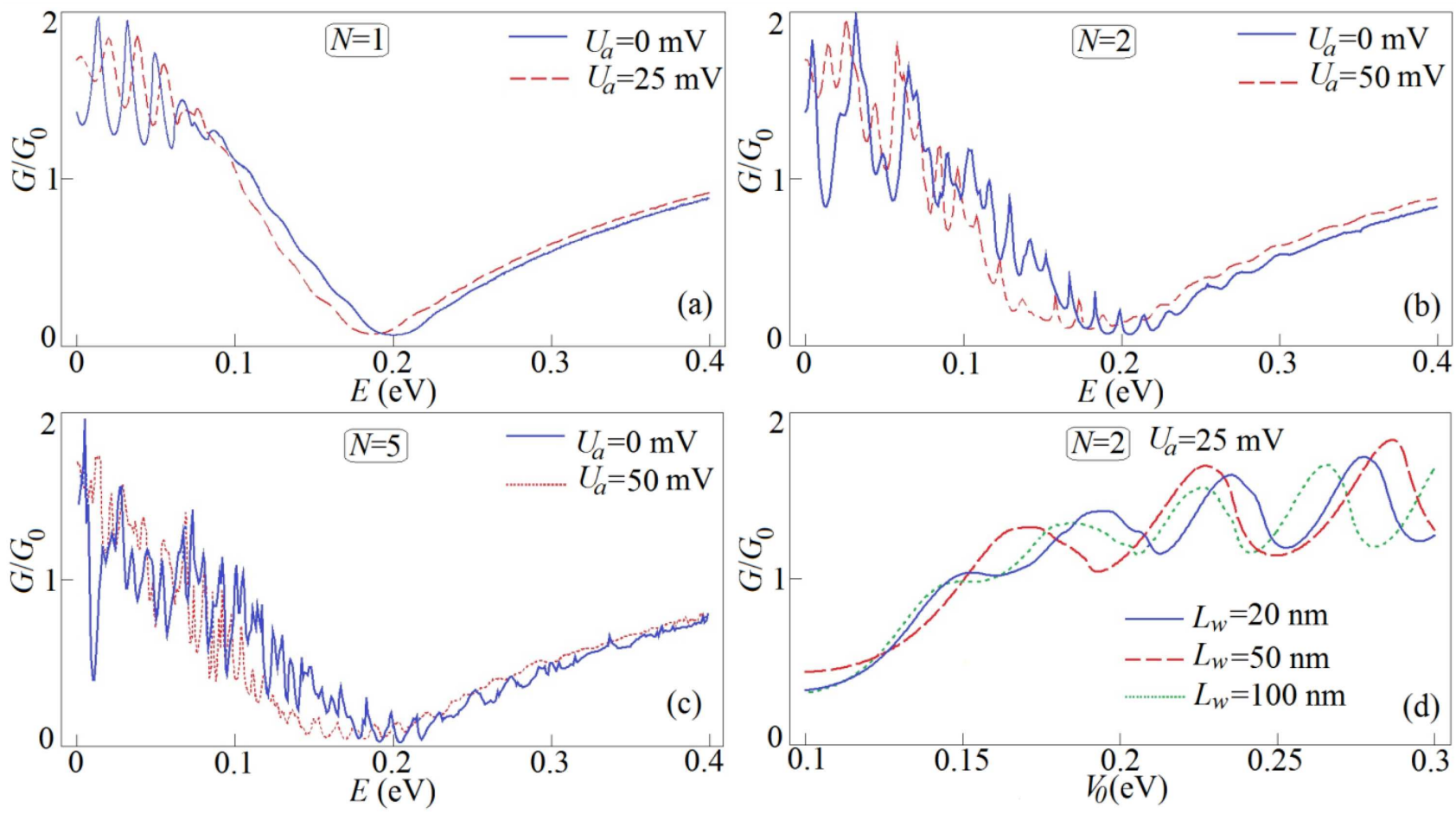

Fig. 6. (a, b, c) Conductance as a function of the incident energy; for $N=1, L_{b}=100 \mathrm{~nm}$; for $N=2, L_{b 1}=50 \mathrm{~nm}$, $L_{w}=100 \mathrm{~nm}, L_{b 2}=70 \mathrm{~nm}$; for $N=5, L_{b 1}=L_{b 3}=L_{b 5}=50 \mathrm{~nm}, L_{b 2}=L_{b 4}=70 \mathrm{~nm}$ and $L_{w}=100 \mathrm{~nm} ;$ (d) conductance as a function of the potential height $V_{0}$, for $N=2, U_{a}=25 \mathrm{mV}$ and three different widths of the well (in this case $L_{b 1}=50 \mathrm{~nm}$ and $L_{b 2}=70 \mathrm{~nm}$ ).

For the unbiased simple barrier structure (solid line in Fig. 6a), at lower energies the conductance exhibits an oscillatory behavior, this being a consequence of the transmission resonances. With increase in energy, a pronounced forbidden domain appears, this being a valley of almost zero conductance, due to the existence of the transmission gap. The forbidden region also appears for $N=2$ and 5 (Fig. $6 \mathrm{~b}$ and c), but the oscillatory behavior of the conductance is more pronounced and the frequency of oscillation increases with the number of barriers (and wells). In addition, certain peaks appear in the valleys, originating from the line-type resonances in the transmission gap. We can see that for all biased structures (dashed and dotted lines in parts (a), (b), and (c), respectively), the conductance profile is shifted to lower energies (due to external electric field) and the amplitude of oscillations is diminished. This behavior is indicated here only for $U_{a}=25 \mathrm{mV}$ (at $N=1$ ) and $U_{a}=50 \mathrm{mV}$ (at $N=2$ and 5), but it is quite clear that the shift for lower energies continues with the increase of the bias voltage.

It must be pointed that the conductance is also an oscillatory function on the barrier height $V_{0}$. This behavior is illustrated in Fig. 6d for the asymmetric double barrier, for an incident energy $E<V_{0}$ (the Klein tunneling region) and a bias voltage $U_{a}=25 \mathrm{mV}$. We can see that the conductance increases with increase in $V_{0}$ and the oscillations can be modified by varying the well width
(20, 50, and $100 \mathrm{~nm}$, respectively). Finally, we mention that our investigations can be extended to other quantum structures based on graphene, for different parameters of the barriers and wells, in external electric or/and magnetic fields.

\section{Conclusions}

Our results are new and highlight the effect of the electric field on various types of asymmetric multi-barrier structures based on monolayer graphene. The interplay between the field-induced asymmetry and the asymmetry of the selected quantum structures could be useful for obtaining a convenient tuning of the transmission in potential applications with graphene-based devices. As we mentioned before, for certain unbiased structures our results agree with those obtained previously by other authors, confirming the accuracy of our numerical calculations. Our numerical analysis clearly reveals that for lower angles of incidence, in certain unbiased asymmetric structures (with two or four wells) the line-type resonant peaks in the transmission gap are not suppressed.

These peaks can be tuned (suppressed or shifted) only for biased structures, using the external field. For all investigated structures, the ballistic conductance presents a forbidden region, due to the existence of the transmission gap. By an adequate adjusting of the structure parameters (the potential height, the number, and the 
lengths of the barriers/wells) the conductance can be controlled using relatively small values of the electric field. Such control of the line-type resonance peaks and of the conductance, respectively, could also be useful in designing resonant tunneling diodes or other high-speed devices based on graphene.

\section{Acknowledgments}

I would like to thank my advisor, Prof. Dr. I. Cotǎescu, for his guidance that he has given me over the past four years.

\section{References}

[1] K.S. Novoselov, A.K. Geim, S.V. Morozov, D. Jiang, Y. Zhang, S.V. Dubonos, I.V. Grigorieva, A.A. Firsov, Science 306, 666 (2004).

[2] A.H. Castro Neto, F. Guinea, N.M.R. Peres, K.S. Novoselov, A.K. Geim, Rev. Mod. Phys. 81 , 109 (2009).

[3] N.M.R. Peres, Rev. Mod. Phys. 82, 2673 (2010).

[4] S. Das Sarma, Shaffique Adam, E.H. Hwang, E. Rossi, Rev. Mod. Phys. 83, 407 (2011).

[5] M.I. Katsnelson, K.S. Novoselov, A.K. Geim, Nat. Phys. 2, 620 (2006).

[6] M.I. Katsnelson, Graphene. Carbon in Two Dimensions, Cambridge University Press, New York 2012.

[7] L.E.F. Foa Torres, S. Roche, J.-C. Charlier, Introduction to Graphene-Based Nanomaterials: From Electronic Structure to Quantum Transport, Cambridge University Press, 2014.

[8] G. Giovannetti, P.A. Khomyakov, G. Brocks, P.J. Kelly, J.V. den Brink, Phys. Rev. B 76, 073103 (2007).

[9] D. Jariwala, A. Srivastava, P.M. Ajayan, J. Nanosci. Nanotechnol. 11, 6621 (2011).
[10] B. Huard, J.A. Sulpizio, N. Stander, K. Todd, B. Yang, D. Goldhaber-Gordon, Phys. Rev. Lett. 98, 236803 (2007).

[11] J.M. Pereira, J.V. Mlinar, F.M. Peeters, P. Vasilopoulos, Phys. Rev. B 74, 045424 (2006).

[12] C. Bai, X. Zhang, Phys. Rev. B 76, 075430 (2007).

[13] J.M. Pereira, P. Vasilopoulos, F.M. Peeters, Microelectron. J. 39, 534 (2008).

[14] N. Stander, B. Huard, D.G. Gordon, Phys. Rev. Lett. 102, 026807 (2009).

[15] Q.S. Wu, S.N. Zhang, S.J. Yang, J. Phys. Condens. Matter 20, 485210 (2008).

[16] M.R. Masir, P. Vasilopoulos, F.M. Peeters, Phys. Rev. B 79, 035409 (2009).

[17] A. Zubarev, D. Dragoman, Physica E 44, 1687 (2012).

[18] J.D. Lu, B. Xu, Mater. Sci. Semicond. Process. 16 , 1008 (2013)

[19] R. Biswas, S. Mukhopadhyay, C. Sinha, Physica E 42 , 1781 (2010).

[20] F. Sattari, J. Theor. Appl. Phys. 9, 81 (2015).

[21] M. Drienovsky, F.X. Schrettenbrunner, A. Sandner, D. Weiss, J. Eroms, M.H. Liu, F. Tkatschenko, K. Richter, Phys. Rev. B 89, 115421 (2014).

[22] X. Chen, J.W. Tao, Appl. Phys. Lett. 94, 262102 (2009).

[23] P.E. Allain, J.N. Fuchs, Eur. Phys. J. B 83, 301 (2011).

[24] L. Sun, Y. Guo, J. Appl. Phys. 109, 123719 (2011).

[25] R. Tsu, L. Esaki, Appl. Phys. Lett. 22, 562 (1973).

[26] W.T. Lu, Y.L. Wang, C.Z. Ye, H. Jiang, W. Li, Physica B 407, 4735 (2012).

[27] Y. Xu, Y. He, Y. Yang, Appl. Phys. A 115, 721 (2014).

[28] C.H. Pham, V.L. Nguyen, J. Phys. Condens. Matter 27, 095302 (2015). 